\title{
TAXONOMIC STUDY ON THE SUBGENUS URESIPEDILUM (DIPTERA: CHIRONOMIDAE: POLYPEDILUM), WITH DESCRIPTION OF A NEW SPECIES FROM THE YAEYAMA ISLANDS, OKINAWA, JAPAN
}

\author{
NAO YAMAMOTO ${ }^{1, *}$, MASARU YAMAMOTO², \\ and TOSHIYA HIROWATARI ${ }^{3}$ \\ ${ }^{1}$ Entomological Laboratory, Graduate School of Life and Environment Science, Osaka Prefecture University, Nakaku Gakuen-cho 1-1, \\ Sakai, Osaka Prefecture, 599-8531 Japan \\ 2 1-6-12, Satomachi, Yoshimi, Shimonoseki, Yamaguchi Prefecture, 759-6525 Japan \\ ${ }^{3}$ Entomological Laboratory, Faculty of Agriculture, Kyushu University, Fukuoka Prefecture, 812-8531 Japan \\ * Corresponding author: nao53210@plant.osakafu-u.ac.jp
}

\section{ABSTRACT}

As a result of a multi-year survey, we recognized four species including a new species of the subgenus Uresipedilum from the Yaeyama Islands, the Ryukyus, Japan. Polypedilum (Uresipedilum) paraconvictum sp. nov. is described. P. (U.) classiglobum Zhang et Wang (2004) P. (U.) bingoparadoxum Kawai et al. (1998) and $P$. (U.) iriofegeum Sasa et Suzuki (2000) are re-described. The first species is newly recorded from Japan, and the second species is new to the Ryukyus. The diagnostic characters of the subgenus are discussed.

Keywords: Uresipedilum, superior volsella, reconfirmation of the diagnosis, Yaeyama Islands

\section{Introduction}

The genus Polypedilum Kieffer, 1912 is one of the largest genera in the family Chironomidae and divided into eight subgenera by Sæther et al. (2010). Hitherto, 127 species including all subgenera and 1 unplaced species have been reported from Japan (Cerobregma Sæther et Sundal: 2 species; Kribionympha Kieffer: 1 species; Pentapedilum Kieffer: 18 species; Polypedilum s. str.: 70 species; Proborum Andersen et Sæther: 1 species; Tripedilum Kieffer: 1 species; Tripodura Townes: 20 species; Uresipedilum Oyewo et Sæther: 12 species; unplaced species: 1 species) (Yamamoto and Yamamoto 2014). The genus Polypedilum is distinguished from the related genera, i.e., Phaenopsectra Kieffer and Sergentia Kieffer, by having deeply bifid pulvilli and the basal constriction of the eighth tergite producing a triangular shape.

The name Uresipedilum was firstly proposed without a description and the taxonomic statement by Sasa and Okazawa (1991). In 1995, Sasa and Kikuchi erected the subgenus Uresipedilum of the genus Polypedilum Kieffer, 1912, with a brief diagnosis and no designation of the type species, and mentioned that this new subgenus is identical to the cultellatum group sensu Sasa (1989) or convictum group sensu Niitsuma (1992). The name Uresipedilum Sasa and Okazawa, therefore, was nomen nudum. In 1998, Oyewo and Sæther (1998) redefined the subgenus Uresipedilum and designated Chironomus convictum Walker, 1856 [= Polypedilum convictum] as the type species. Then, the subgenus Uresipedilum was redefined with a slight change by Sæther et al. (2010) as follows: the adult males have the basal portion of the superior volsella much longer than wide without prominent inner projection and wing membrane without markings or setae; the pupae are not distinguishable from those of Polypedilum s. str., Pentapedilum Kieffer and Problum Andersen et Sæther; the larvae have well developed to at least posterior lobes on the ventromental plates. Polypedilum (Uresipedilum) bullum Zhang et Wang (2004) was removed from the subgenus Uresipedilum and treated as belonging to the subgenus Probolum by their diagnosis. Therefore, this subgenus has been defined by a part of character of the adult and larvae at present.

During a multi-year survey, we have continued to clarify the chironomid fauna in the Ryukyus, especially the Yaeyama Islands, located in the subtropical region in the southernmost area of Japan, between Taiwan and Okinawa Island. As a result, we recognized four species belonging to the subgenus Uresipedilum Oyewo et Sæther from this area. Of these species excluding $P$. (U.) iriofege$u m$, one is new to science, one is new to Japan and one is new to this area. Here, we give the description of these four species and discuss about the diagnostic characters of the subgenus Uresipedilum in the following lines.

\section{Materials and Methods}

Description and re-descriptions of coloration were made on the basis of dried specimens. Before being described and illustrated, the dried specimens were macerated in a 5\% hot $\mathrm{KOH}$ solution for 3-5 minutes. After relaxing, they were rinsed in distilled water containing a small quantity of glacial acetic acid, and then dissected with micro-pins in glycerin. The specimens used for description, re-descriptions and illustrations were then 
mounted permanently on slide in Canada balsam, while other specimens were preserved in dry condition. In the description and re-descriptions, the measurements are given as ranges, followed by the number of species measured in parentheses (n). Body length and wing length are indicated in millimeters, remaining lengths of character are indicated in micrometer.

The type specimen of Polypedilum iriofegeum was borrowed from the National Museum of Nature and Science, Tokyo (NSMT) and was used for illustration. Polypedilum bingoparadoxum was borrowed from Dr. K. Kawai of Hiroshima University.

The morphological nomenclature mainly follows Sæther (1980) with the modifications and additions given in Sæther (1990). With the labium, the term prementum is used in accordance with the usage of Hoyt (1952).

Most of the specimens examined are housed in the Entomological Laboratory, Osaka Prefecture University (OPU), Sakai, Osaka, Japan.

\section{Taxonomy}

\section{Polypedilum (Uresaipedilum) paraconvictum sp. nov.}

(Figs. 1a-c)

\section{Type material}

Holotype: male (No. OPU-NY167), Japan, Ryukyus, Okinawa Pref., Yaeyama Isls., Iriomote Is., Funaura, 10. IV. 1999, M. Yamamoto; Paratypes: 5 males, as Holotype except 21. III. 2000.

\section{Etymology}

From the Latin, para, resemble and convictum, referring to its resemblance to Polypedilum convictum (Walker in morphological characters in the male adult.

\section{Male $(n=4)$}

Total length $1.9 \mathrm{~mm}$. Wing $1.6 \mathrm{~mm}$ long, $0.35 \mathrm{~mm}$ wide; wing length/wing width 4.6.

Coloration: Head yellowish brown in ground color; antennal flagellum pale brown. Thorax yellowish brown. Legs yellow. Halter yellowish white. Abdomen yellow.

Head: Frontal tubercle absent. Vertex with 9 temporal setae. AR 0.97-1.05. Prementum with 3 setae. Palpomere

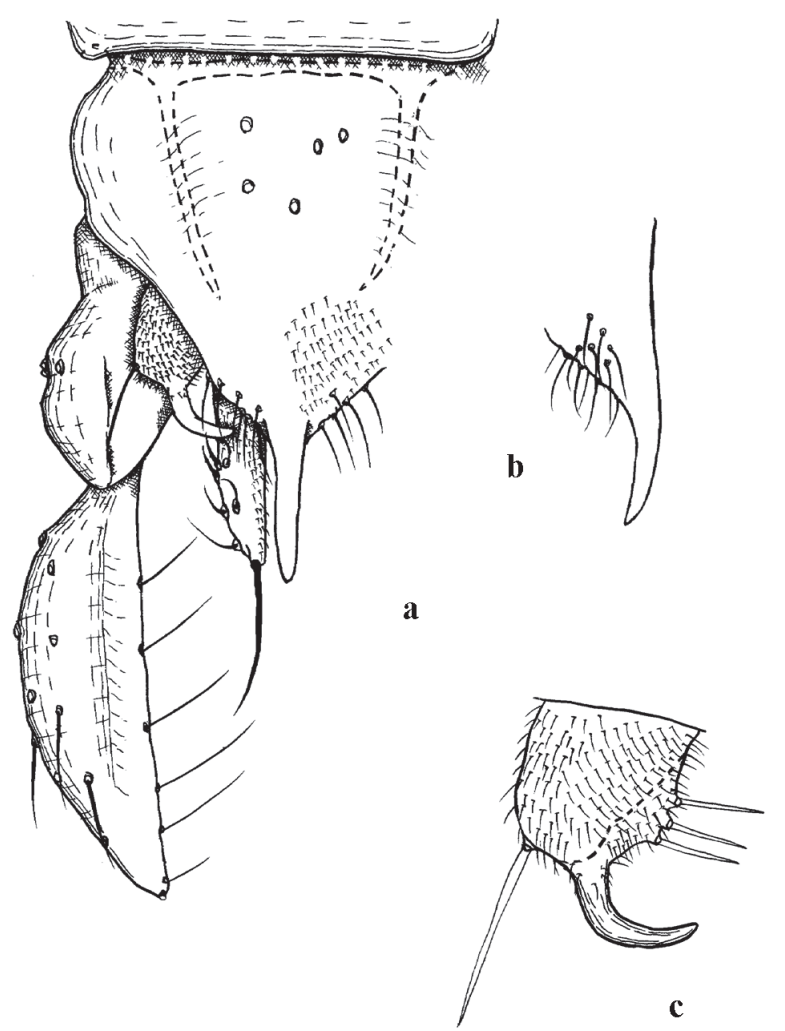

Fig. 1 Adult male of Polypedilum (Uresipedilum) paraconvictum sp. nov. a: hypopygium, dorsal view; b: anal point, lateral view; c: superior volsella.

lengths (in $\mu \mathrm{m}$ ): $25,35,85,105,140$; with $3,3,11,10,6$ setae, respectively; 3 rd palpomere with 1 sensilla clavata. Clypeus with 9 setae.

Thorax: Lateral antepronotals absent. Dorsocentrals 11, uniserial, including 3 humerals; acrostichals 13, biserial; prealars 3, uniserial. Scutellum with 5 setae, uniserial.

Wing: Anal lobe weakly developed. VR 1.20. $\mathrm{R}_{2+3}$ adjacent to $R_{1} \cdot R, R_{1}$ and apical $2 / 3$ of $R_{4+5}$ with $14,7,16$ setae, respectively. Brachiolum with 1 seta. Squama with 3 setae.

Legs: Scale of fore tibia triangular, rounded, without apical short spur. Lengths (in $\mu \mathrm{m}$ ) and proportions of legs as in Table 1.

Hypopygium (Figs. 1a-c): Tergum IX with 8 median setae. Anal point long, slender, parallel-sided, with rounded apex in dorsal view; narrowed, parallel-sided,

Table 1 Lengths (in $\mu \mathrm{m}$ ) and proportions of legs of Polypedilum (Uresipedilum) paraconvictum sp. nov. Male $(\mathrm{n}=4)$.

\begin{tabular}{|c|c|c|c|c|c|c|c|c|c|c|}
\hline & fe & ti & $\operatorname{ta}_{1}$ & $\operatorname{ta}_{2}$ & $\operatorname{ta}_{3}$ & $\operatorname{ta}_{4}$ & $\operatorname{ta}_{5}$ & LR & BV & SV \\
\hline$p_{1}$ & $620-760$ & $420-440$ & - & 820 & 570 & 360 & 160 & - & - & - \\
\hline$p_{2}$ & $680-720$ & $570-610$ & $320-355$ & 180-190 & $120-140$ & $90-100$ & $50-55$ & $0.56-0.62$ & $3.41-3.51$ & $3.61-3.91$ \\
\hline$p_{3}$ & $760-780$ & $610-640$ & $430-420$ & $260-280$ & $220-240$ & $130-160$ & $60-70$ & $0.67-0.70$ & 2.40.-2.70 & $3.19-3.31$ \\
\hline
\end{tabular}

* p1-3: front-, mid-, hind-legs. fe: femur. ti: tibia. $\mathrm{ta}_{1}, \mathrm{ta}_{2}, \ldots, \mathrm{ta}_{5}$ : tasomeres 1-5. LR: leg ratio. BV: combined length of femur, tibia and basitarsus divided by combined length of tarsomeres $2-5$. SV: ratio of femur plus tibia to metatarsus. 
Table 2 Lengths (in $\mu \mathrm{m}$ ) and proportions of legs of Polypedilum (Uresipedilum) classiglobum Zhang et Wang male $(\mathrm{n}=3)$.

\begin{tabular}{|c|c|c|c|c|c|c|c|c|c|c|}
\hline & fe & ti & $\mathrm{ta}_{1}$ & $\mathrm{ta}_{2}$ & $\mathrm{ta}_{3}$ & $\mathrm{ta}_{4}$ & $\mathrm{ta}_{5}$ & LR & BV & SV \\
\hline$p_{1}$ & $890-970$ & $650-710$ & $1040-1120$ & $770-790$ & 560 & $470-500$ & $200-210$ & $1.58-1.60$ & $1.29-1.36$ & $1.48-1.50$ \\
\hline$p_{2}$ & 910-1010 & $820-890$ & $460-510$ & $270-280$ & $200-220$ & $130-140$ & $60-80$ & $0.53-0.62$ & $3.29-3.39$ & $3.39-4.04$ \\
\hline $\mathbf{p}_{3}$ & 1040-1110 & $930-940$ & $730-770$ & $380-400$ & $330-350$ & $200-210$ & $90-100$ & $0.78-0.83$ & $2.65-2.71$ & $2.56-2.81$ \\
\hline
\end{tabular}

pointed apically in lateral view. Superior volsella with basal portion well developed, covered with microtrichia, with 3 inner setae, with 1 long seta on its outer corner, and with apical projection strongly curved medially, nearly as long as basal portion. Inferior volsella long, parallel-sided, with 8 setae on its apical 1/3 of which the apical one is long and extending posteriorly. Sternapodeme narrow and anterior margin nearly as wide as gonocoxite width. Gonocoxite with 4 uniserially arranged setae on inner margin. Gonostylus long, semicircular in shape, with one short apical and 6 long setae on inner margin of apical $4 / 5$.

\section{Remarks}

The species is very similar to Polypedilum (Polypedilum) convictum (Walker 1856). However, it is distinguished from the latter by having the apical projection of superior volsella which is strongly curved inwardly at middle and low value AR.

\section{Distribution}

Japan (Yaeyama Islands: Iriomote Island).

\section{Polypedilum (Uresipedilum) classiglobum Zhang et Wang, 2004}

(Figs. 2a-d)

Polypedilum (Uresipedilum) classiglobum Zhang et Wang, 2004: 12.

\section{Material examined}

4 males, Japan, Ryukyus, Okinawa Pref., Yaeyama Isls., Iriomote Is., Kampira Fall, 18. II. 2003, M. and N. Yamamoto.

\section{Male $(n=4)$}

Total length 3.1-3.3 mm. Wing 1.8-1.9 mm long, 0.50-0.524 mm wide; wing length/wing width 3.4-3.9

Coloration: Head yellowish brown in ground color; antennal flagellum pale brown. Thorax yellowish brown; scutellum whitish. Legs yellowish brown in ground color; tibiae, fore tibia concentrated (?). Halter yellowish white. Abdominal segments yellowish brown or pale green.

Head: Frontal tubercle absent. Vertex with 12-13 temporal setae. AR 1.20-1.43. Prementum with 1 seta. Clypeus with 12-14 setae.

Thorax: Lateral antepronotals absent. Dorsocentrals 14-17, including 2 humerals, uniserial; acrostichals 10-12, biserial; prealars 4, uniserial. Scutellum with 10-11 setae, biserial.
Wing: Anal lobe moderately developed. VR 1.23-1.36. $R_{2+3}$ nearly parallel to $R_{1} . R, R_{1}$ and $R_{4+5}$ with $19-21$, 17-21, 31-36 setae, respectively. Squama with 7 setae.

Legs: Scale of fore tibia triangular, rounded apex. Lengths (in $\mu \mathrm{m}$ ) and proportions of legs as in Table 2.

Hypopygium (Figs. 2a-d): Tergum IX with 9-10 median setae. Anal point long, slender, parallel-sided, with rounded apex in dorsal view; stout, horn- or sickle-shaped in lateral view. Superior volsella with basal portion well-developed, wide, nearly rectangular, with 5 setae on tis inner margin, with a long apical seta on its outer corner; with projection variable in shape, length and its direction as in Figs. 2a,c,d. Inferior volsella well-developed, gradually tapering to apex, with 18-20 setae

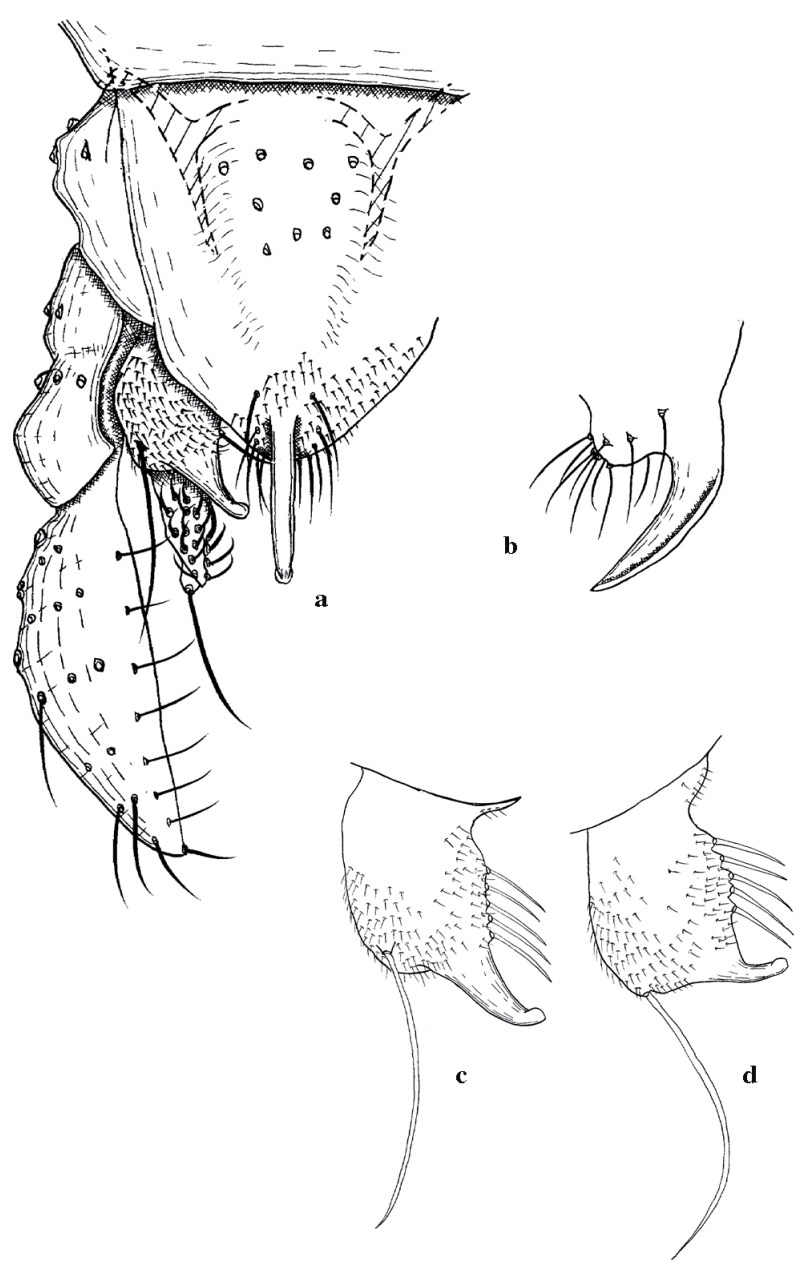

Fig. 2 Adult male of Polypedilum (Uresipedilum) classiglobum Zhang et Wang. a: hypopygium, dorsal view; b: anal point, lateral view; c, d: superior volsella. 
on apical $1 / 2$ of which the apical one is long and extending posteriorly. Sternapodeme narrow, without anterolateral projection, anterior margin nearly as wide as gonocoxite width. Gonocoxite with 4-5 uniserially arranged setae on inner margin. Gonostylus long, semicircular in shape, with one short apical and 7-11 inner setae.

\section{Remarks}

This species is distinct from any other species of the subgenus Uresipedilum by having the well-developed and nearly rectangular basal portion of superior volsella with 5 inner setae and horn- or sickle-shaped anal point in lateral view. This species is new to Japan, and was collected on the banks of clean streams as in the Yaeyama Islands.

\section{Distribution}

China (Oriental Region), Japan (Yaeyama Is.: Iriomote Is.)

\section{Polypedilum (Uresipedilum) iriofegeum Sasa et Suzuki}

(Figs. 3a-b)

Polypedilum (Polypedilum) iriofegeum Sasa et Suzuki, 2000: 15 .

\section{Material examined}

Holotype: male [NSMT, No. 385:74] Japan, Ryukyus, Okinaw Pref., Yaeyama Isls., Iriomote Is., Kuira River, H. Suzuki; 3 males, as previous except Ootomi, 5. VII. 1992, light trap, K. Ohara.

\section{Male $(n=4)$}

Total length $2.3 \mathrm{~mm}$. Wing $1.45 \mathrm{~mm}$ long, $0.43 \mathrm{~mm}$ wide; wing length/wing width 3.4 .

Coloration: Head dark brown in ground color; antennal flagellum pale brown; antennal pedicel, maxillary pulpus brown. Thorax blackish brown. Legs predominantly yellowish brown; all coxae blackish brown; fore tibia brown except for apex. Abdomen blackish brown.

Head: Frontal tubercle absent. Vertex with 9 temporal setae. AR 0.78. Clypeus with 21 setae.

Thorax: Lateral antepronotals absent. Dorsocentrals 14 uniserial, including 4 humerals; acrostichals 13 , biserial; prealars 4, uniserial. Scutellum with 8 setae, biserial.

Wing: Anal lobe moderately developed. VR 1.33. $\mathrm{R}_{2+3}$ nearly parallel, separated from $\mathrm{R}_{1}$. Squama with 10 setae.

Legs: Scale of fore tibia triangular, with terminal extension. Lengths (in $\mu \mathrm{m}$ ) and proportions of legs as in Table 4.

Hypopygium (Figs. 1a-c): Tergum IX with 16 median setae. Anal point long, slender, parallel-sided, with pointed apex in dorsal view. Superior volsella with apical projection long, gradually curved medially, gently tapering to rounded apex, with basal portion rectangular in shape, covered with microtrichia, with a dorsolateral seta on its outer corner and with 2 inner setae. Inferior volsella long, parallel-sided, not beyond the tip of anal point, with 12 recurved setae on its apical $1 / 3$ of which the apical one is long and extending posteriorly. Sternapodeme narrow. Gonocoxite with 4 uniserially arranged setae on inner margin. Gonostylus long, slender, with one short apical and 4 long setae on inner margin of apical 2/3.

\section{Remarks}

This species is distinct from any other Uresipedilum species by having the elongate projection and the well-developed nearly quadrate basal portion with 2 inner setae in the superior volsella.

\section{Distribution}

Japan (Yaeyama Islands: Iriomote Island)

\section{Polypedilum (Uresipedilum) bingoparadoxum Kawai, Inoue et Imabayashi}

(Fig. 3c)

Polypedilum (Uresipedilum) bingoparadoxum Kawai, Inoue et Imabayashi, 1998: 378

\section{Material examined}

Type material: Holotype, male, Japan, Hiroshima Prefecture, Shin-ichi, Fujio, Kaya River, 9. Xi. 1994, K. Kawai; 1 male, Japan, Ryukyus, Okinawa Pref., Yaeyama Isls., Iriomote Is., Funaura, 19. XI. 2001, N. Yamamoto. (No. OPU-NY84).

\section{Male $(n=1)$}

Total length $2.4 \mathrm{~mm}$. Wing $1.6 \mathrm{~mm}$ long, $0.5 \mathrm{~mm}$ wide; wing length/wing width 3.2.

Coloration: Head yellow in ground color; antennal flagellum, clypeus, palpomeres brown. Mesonotum except pale brown postnotum yellow. Legs yellow in ground color. Abdomen predominantly yellow.

Head: Frontal tubercle absent. Vertex with 8 temporal setae. AR 1.5. Prementum with 4 setae. Palpomere lengths (in $\mu \mathrm{m}$ ): $33,40,78,80,138$; with $2,5,16,14,6$ setae, respectively; third palpomere with 5 sensilla clavata. Clypeus with 12 setae.

Thorax: Lateral antepronotals absent. Dorsocentrals 14, including 5 humerals, uniserial; acrostichals 14, biserial; prealars 5, uniserial. Scutellum with 3 setae, uniserial.

Wing: Anal lobe moderately developed. VR 1.3. $\mathrm{R}_{2+3}$ nearly parallel with $R_{1}$ and ended at basal $1 / 6$ between apices of $\mathrm{R}_{1}$ and $\mathrm{R}_{4+5} . \mathrm{R}, \mathrm{R}_{1}$ and apical $2 / 3$ of $\mathrm{R}_{4+5}$ with 21 , 15,28 setae, respectively. Squama with 12 setae.

Legs: Scale of fore tibia triangular, with smoothly rounded apex, with a tiny apical spine; fore, mid and hind trochanters with 7, 11, 7 marginal setae, respectively. Lengths (in $\mu \mathrm{m}$ ) and proportions of legs as in Table 3.

Hypopygium (Fig. 3c): Tergum IX with 5 median setae. Anal point long, robust, parallel-sided, with rounded apex. Superior volsella robust, basal $1 / 2$ sparsely covered with microtrichia and apical $1 / 2$ bare, apical portion abruptly narrowed and hooked inwardly and anteriorly, basal portion with 2-3 inner setae and a dorsolateral seta inserted at about basal 1/3. Inferior volsella long, well de- 
Table 3 Lengths (in $\mu \mathrm{m}$ ) and proportions of legs of Polypedilum (Uresipedilum) bingoparadoxum Kawai et al. male $(\mathrm{n}=1$ ).

\begin{tabular}{|c|c|c|c|c|c|c|c|c|c|c|}
\hline & fe & ti & $\mathrm{ta}_{1}$ & $\mathrm{ta}_{2}$ & $\mathrm{ta}_{3}$ & $\mathrm{ta}_{4}$ & $\mathrm{ta}_{5}$ & LR & BV & SV \\
\hline$p_{1}$ & 720 & 460 & - & - & - & - & - & - & - & - \\
\hline$p_{2}$ & 740 & 520 & 340 & 190 & 160 & 110 & 60 & 0.65 & 3.08 & 3.71 \\
\hline$p_{3}$ & 770 & 720 & 370 & 310 & 240 & 170 & 90 & 0.51 & 2.3 & 4.03 \\
\hline
\end{tabular}

Table 4 Lengths (in $\mu \mathrm{m}$ ) and proportions of legs of Polypedilum (Uresipedilum) iriofegeum Sasa et Suzuki male $(\mathrm{n}=4)$.

\begin{tabular}{ccccccccccc}
\hline & $\mathbf{f e}$ & $\mathbf{t i}$ & $\mathbf{t a}_{\mathbf{1}}$ & $\mathbf{t a}_{\mathbf{2}}$ & $\mathbf{t a}_{\mathbf{3}}$ & $\mathbf{t a}_{\mathbf{4}}$ & $\mathbf{t a}_{\mathbf{5}}$ & $\mathbf{L R}$ & $\mathbf{B V}$ & \\
\hline $\mathbf{p}_{\mathbf{1}}$ & $610-670$ & $410-430$ & $810-830$ & $590-620$ & $360-390$ & $260-280$ & $110-130$ & $1.90-1.98$ & $1.38-1.41$ & $1.30-1.33$ \\
$\mathbf{p}_{\mathbf{2}}$ & $710-790$ & $590-650$ & $290-350$ & $200-230$ & $130-160$ & $80-100$ & $50-60$ & $0.49-0.54$ & $3.13-3.46$ & $4.03-4.48$ \\
$\mathbf{p}_{\mathbf{3}}$ & $740-830$ & $670-700$ & $480-560$ & $280-320$ & $220-280$ & $160-170$ & $70-80$ & $0.72-0.82$ & $2.42-2.59$ & $2.59-2.93$ \\
\hline
\end{tabular}

veloped, with 11 recurved setae on its apical 1/2. Sternapodeme narrow. Gonocoxite with 4 uniserially arranged setae on inner margin. Gonostylus long, moderately developed, with one short apical seta and 5-6 long setae on inner margin of apical 3/5.

\section{Remarks}

This species is distinguished from other members of the subgenus Polypedilum by having the robust superior volsella of which the projection is abruptly narrowed apically and hooked inwardly and anteriorly. Kawai et al. (1998) assigned this species to the subgenus Uresipedi-
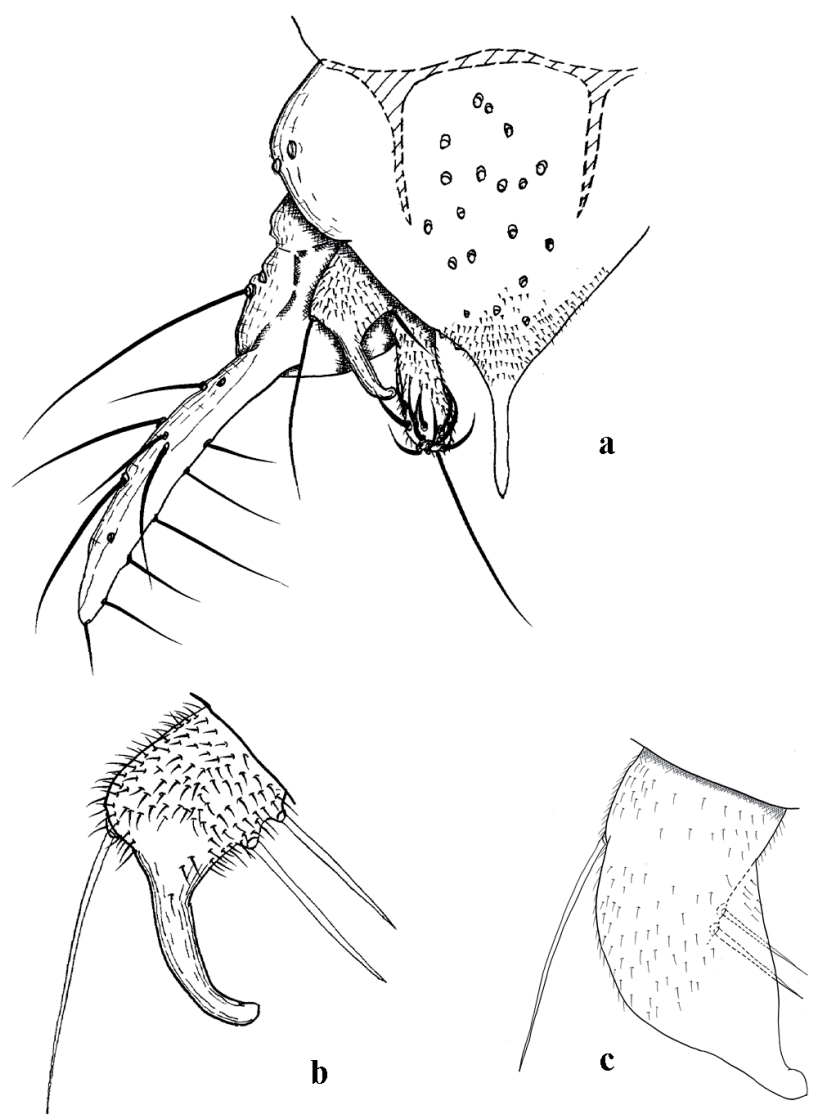

Fig. 3 Adult male of Polypedilum (Uresipedilum) iriofegeum Sasa et Suzuki (a-b) and Polypedilum (Uresipedilum) bingoparadoxum Kawai (c) a: hypopygium, dorsal view; b-c: superior volsella. lum, although the reason is not given. Sæther and Oyewo (2008) supported their treatment as having the projection of the superior volsella is shorter than $1 / 3$ length of the basal potion. However, the projection recognized by them is indicated as a part of whole projection. The projection of this species is well developed and is as long as, or longer than basal portion. This species is newly recorded from the Ryukyus (Oriental region), and was collected on the banks of clean streams as in the Yaeyama Islands.

\section{Distribution \\ Japan (Hiroshima Pref. and Yaeyama Islands)}

\section{Discussion}

With regard to the adult diagnosis of Uresipedilum, Oyewo and Sæther (1998) and Sæther and Oyewo (2008) followed the statement in the "key to subgenera and groups" of Sasa and Kikuchi (1995: 112). And, Sæther et al. (2010) also followed Sasa and Kikuchi (1995). In their phylogenetic analysis, the well-developed basal portion and relative length of apicomedial projection to the basal portion of the superior volsella are important features for assigning the subgenus Uresipedilum. The character states selected for their phylogenetic analysis are as follows: characters number 14-18 in Oyewo and Sæther (1998) and Sæther and Oyewo (2008), 18-20, 23, 24 and 28 in Sæther et al. (2010). In Sæther et al. (2010), it seems to be a most important taxonomic character and character state (character state 1 of character number 20) for inferring the monophyly of the subgenus Uresipedilum the apicomedial projection of which is much shorter than the basal portion in the superior volsella. This character and character state 1 of character 19 are inferred the synapomorphies of Probolum and Uresipedilum. However, Probolum is separable from the latter by having an additional inner lobe in the base of the superior volsella.

In pupae, Uresipedilum is not distinguishable from Polypedilum s. str., Pentapedilum and Probolum (Sæther 
et al. 2010). In larvae, judging from the matrix in appendix 1 given by Sæther et al (2010), it seems that character 62 and 65 are rather important for inferring the monophyly of the subgenus Uresipedilum. The character 62 shows three different states of the ventromental plates. The matrix shows that all nominated species except $P$. (U.) surugense have the ventomental plates with moderate to well-developed posterior lobes. However, Niitsuma (1992) shows surugense with the well-developed posterior lobe in his figures. This character state also is held in common with some species of Tripodura. Although the larvae of Stictochironomus and Phaenopsectra have the ventromental plate with distinct posterior lobe, their matrix indicates these genera have no posterior lobes. The character 65 shows the developmental degree of the medina ends of ventromental plates. In the matrix of appendix 1, all mentioned species except $P$. (U.) surugense have the median ends which are produced anteriorly and in contact with the first lateral teeth. However, judging from the drawings of Sæther and Oyewo (2008) and Niitsuma (1992), this character state is observable only in $P$. (U.) dossenudum and also recognizable in Endochironomus, Nilothuama, Phaenopsectra, Sergentia and Stictochironomus. Therefore, it is difficult to treat the above-mentioned larval characters as the diagnostic characters supporting the monophyly of the subgenus Uresipedilum.

Consequently, as stated above, the subgeneric independence of Uresipedilum is basically secured by the character state of the superior volsella in the male hypopygium. However, some problems are present in treating Uresipedilum as a monophyletic subgenus. For example, Polypedilum (Cerobregma) paucisetum Zhang et al., 2006 has the superior volsella being basically the same as that of Uresipedilum in structure. In some species, it is difficult to recognize the boundary between the basal portion and apicomedial projection. Therefore, further study might serve to elucidate the taxonomic and phylogenetic significance of the superior volsella in the subgenus Uresipedilum.

\section{Acknowledgements}

We are grateful to Dr. A. Shinohara (NSMT) for the loan of the specimens housed in the Sasa Collection and also to Dr. Kawai of Hiroshima University. We wish to express cordial thanks to Mr K. Ohara (Tokushima Prefecture) for providing us with valuable samples. The senior author wishes to express his thanks to Prof. M. Ishii and
Assoc. Prof. N. Hirai (OPU) for their valuable suggestion and advice. Cordial thanks are also due to two anonymous reviewers for their valuable advice.

\section{REFERENCES}

Hoyt P (1952) The evolution of the mouth parts of adult Diptera: Microent 17: 61-125.

Kawai K, Inoue E, Imabayashi H (1998) Seven new species of the genus Polypedilum (Diptera: Chironomidae) from Japan. Jpn J Limnol 59: 367-381.

Niitsuma H (1992) The Polypedilum convictum species group (Diptera, Chironomidae) from Japan, with descriptions of two new species: Jpn J Entomol 60: 693-706.

Oyewo EA, Sæther OA (1998). Revision of Afrotropical Polypedilum Kieffer subgen. Ureshipedilum Sasa et Kikuchi, 1995 (Diptera: Chironomidae) with a review of the subgenus: Ann Limnol 34: 315-362.

Sæther OA (1980) Glossary of chironomid morphology terminology (Chironomidae: Diptera): Entomol Scand Suppl 14: $1-51$.

Sæther OA (1990) A review of the genus Limnophyes Eaton from the Holarctic and Afrotropical regions (Diptera: Chironomidae, Orthocladiinae): Entomol Scand Suppl 35: 1-139.

Sæther OA, Andersen T, Pinho LC, Mendes HF (2010) The problem with Polypedilum Kieffer (Diptera: Chironomidae), with the description of Probolum subgen. n. Zootaxa 2497: $1-36$.

Sæther OA, Oyewo EA (2008) Keys, phylogenies and biogeography of Polypedilum subgen: Uresipedilum Oyewo et Sæther (Diptera: Chironomidae). Zootaxa 1806: 1-34.

Sasa M (1989) Chironomidae of Japan: Checklist of species recorded, key to males and taxonomic notes: Res Rep NIES No 125: $1-177$.

Sasa M, Kikuchi M (1995) Chironomidae (Diptera) of Japan: University of Tokyo press, Tokyo.

Sasa M, Okazawa T (1991) Part 1. Studies on the chironomids of the Joganji River, Toyama (Diptera, Chironomidae). In: The chironomids of the Joganji River and other places. Res Rep TPEP 1991: 52-67.

Sasa M, Suzuki H (2000) Studies on the chironomid species collected on Ishigaki and Iriomote Islands, southern Japan. Trop Med 42: 1-37.

Yamamoto M, Yamamoto N (2014) Family Chironomidae. In: Editorial Committee of Catalogue of the Insect of Japan (ed) Catalogue of the Insects of Japan. Volume 8, part 1 Diptera (Nematocera-Brachcera Aschiza). Touka Shobo, Fukuoka, Japan, pp. 237-362.

Zhang R, Wang X (2004) Polypedilum (Uresipedilum) Oyewo and Sæther from China (Diptera: Chironomidae). Zootaxa 565: $1-38$.

Zhang R, Wang X, Sæther OA (2006) Two unusual species of Polypedilum Kieffer (Diptera: Chironomidae) from Oriental China. Zootaxa 1982: 39-48. 